\title{
Novel deletion of SPAST in a Chinese family with hereditary spastic paraplegia
}

\author{
Yapei Feng ${ }^{1, *}$, Ms, Xin Ke ${ }^{2, *}$, MD, Meng Zhai ${ }^{1}$, Ms, Qian Xin ${ }^{1}$, Ms, Yaoqin Gong ${ }^{1}$, PhD, Qiji Liu ${ }^{1}$, PhD
}

INTRODUCTION Hereditary spastic paraplegia (HSP) belongs to a large, heterogeneous group of progressive neurodegenerative diseases characterised by progressive lower extremity weakness and spasticity, which is caused by developmental failure or degeneration of motor axons in the corticospinal tract. Classical genetic studies have identified at least 46 genetic loci responsible for HSP.

METHODS A genetic study was conducted on a four-generation Chinese family with autosomal dominant HSP. The SPAST gene was investigated using linkage analysis and direct sequencing. Findings were compared with unaffected family members and 50 normal, unaffected individuals who were matched for geographical ancestry.

RESULTS We identified a novel 14-bp heterozygous deletion that induced a frameshift mutation in exon 15 of SPAST (SPG4). This mutation is predicted to have functional impact and found to cosegregate with the disease phenotype.

CONCLUSION Our results have expanded the mutation spectrum of the SPAST gene. These findings could help clinicians provide prenatal diagnosis of affected foetuses in families with a known history of such neurodegenerative diseases.

Keywords: hereditary spastic paraplegia, mutation, spastin, SPG4

\section{INTRODUCTION}

Hereditary spastic paraplegia (HSP) belongs to a highly heterogeneous group of neurodegenerative disorders and is characterised by progressive spasticity and weakness of the lower limbs. The main pathological changes associated with the disorder are developmental failure or degeneration of motor axons in the corticospinal tract. ${ }^{(1,2)}$ Genetic studies have shown the extremely heterogeneous nature of HSP, and at least 46 genetic loci responsible for HSP have been identified in families with autosomal dominant (AD), autosomal recessive and X-linked inheritances, according to the HUGO Gene Nomenclature and OMIM (Online Mendelian Inheritance in Man) databases. ${ }^{(3-5)}$ HSPs can be classified into either pure or complicated forms, based on the clinical manifestations. The more common pure form of HSP usually presents four core features: (a) slowly progressive spastic gait; (b) increasing muscle tone in the lower limbs; (c) hyperreflexia; and (d) extensor plantar response. ${ }^{(6,7)}$ Complicated HSP manifests other neurological or extraneurological abnormalities, such as mental retardation, deafness, cerebellar ataxia, epilepsy, dysarthria and ichthyosis. Although patients with pure HSP generally exhibit the disease's main clinical features, considerable variation is observed in the age of the patient at disease onset, as well as in the rate of progression and severity of spasticity within and between families. ${ }^{(1,2,6,8)}$

The SPG4 gene, which has been mapped to chromosome $2 \mathrm{p} 21-\mathrm{p} 22$, is the most common causative gene of autosomal dominant hereditary spastic paraplegia (ADHSP). Spastic paraplegia type 4 (SPG4) accounts for approximately $40 \%$ of AD pedigrees and $6 \%-15 \%$ of sporadic cases. ${ }^{(9)}$ To date, more than

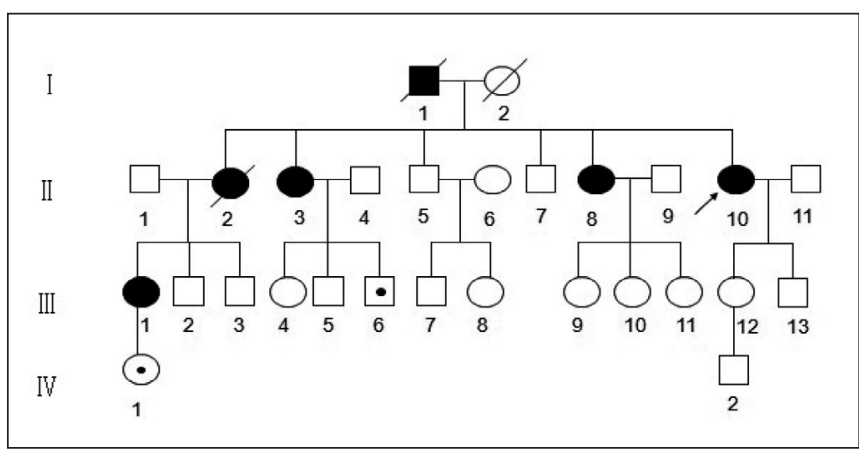

Fig. 1 Pedigree of the four-generation Chinese family with hereditary spastic paraplegia.

257 SPAST mutations have been reported, ${ }^{(10)}$ including missense, nonsense or splice site mutations, small insertions or deletions, and large duplications or deletions. ${ }^{(11-14)}$ SPG4 encodes spastin, a 616 amino acid protein, which is a member of the AAA cassette (adenosine triphosphatases associated with diverse cellular activities). Spastin is a microtubule-severing enzyme ${ }^{(15)}$ involved in remodelling protein complexes with adenosine triphosphate (ATP) hydrolysis and coordination of axonal microtubule interactions with the tubular endoplasmic reticulum network. ${ }^{(16,17)}$ In this context, we conducted a genetic study of a four-generation Chinese family with ADHSP, investigating the SPAST gene via linkage analysis and direct sequencing methodologies.

\section{METHODS}

The patients were from a Chinese family spanning four generations from Weihai, Shandong Province, China. HSP followed a pattern of $A D$ inheritance in this family (Fig. 1). The genetic

\footnotetext{
${ }^{1}$ Key Laboratory for Experimental Teratology of the Ministry of Education and Department of Medical Genetics, Shandong University School of Medicine, Jinan, ${ }^{2}$ Department of Orthopaedics, Hospital of Weihai Economic and Technical Development Zone, Weihai, People's Republic of China

${ }^{*}$ These two authors contributed equally as first author in this work.

Correspondence: Dr Qiji Liu, Professor, Key Laboratory for Experimental Teratology of the Ministry of Education and Department of Medical Genetics, Shandong University School of Medicine, Jinan 250012, People's Republic of China. liuqiji@sdu.edu.cn
} 
Table I. Clinical features of family members with autosomal dominant hereditary spastic paraplegia.

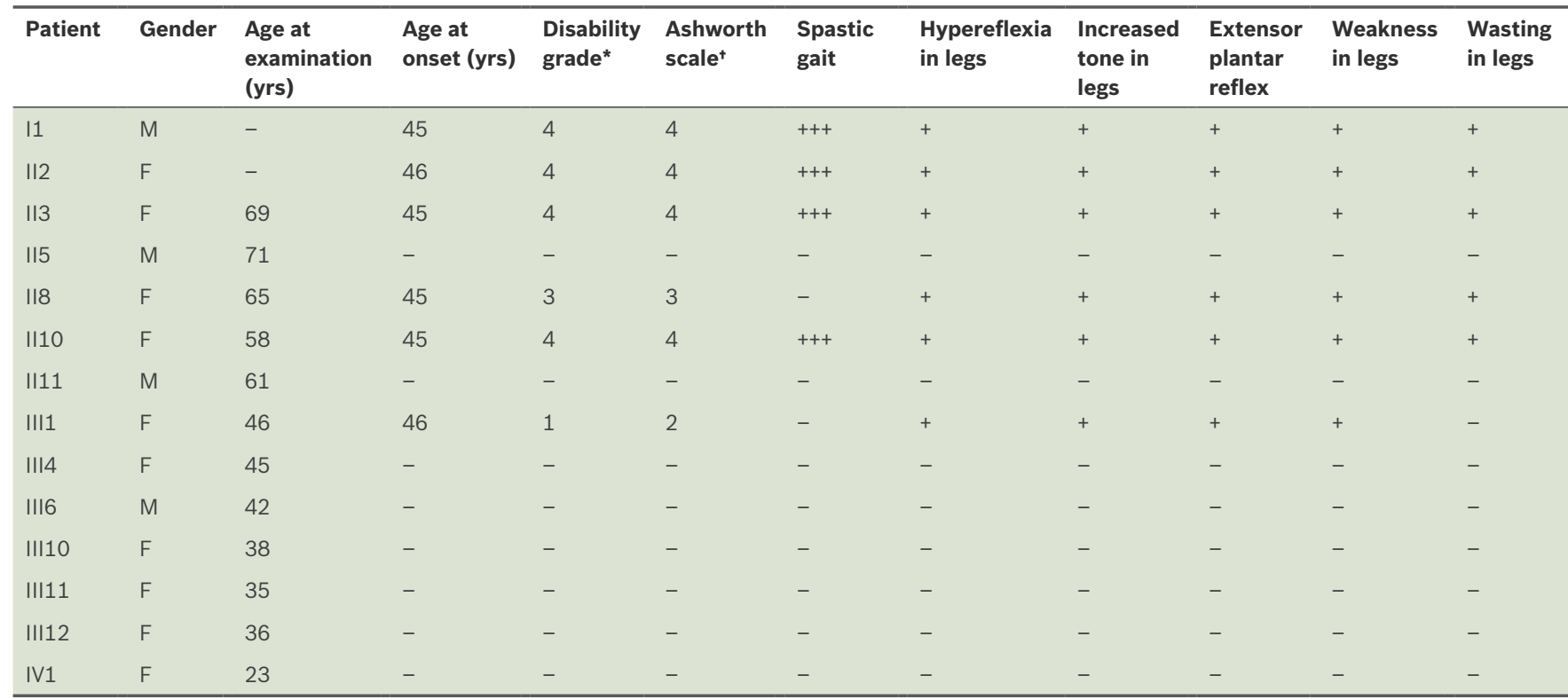

*Disability grades: no mobility problems or slight stiffness of the legs (grade 1); moderate gait stiffness (grade 2); problems running but able to walk alone (grade 3); problems walking (grade 4); wheelchair-bound (grade 5). ${ }^{\dagger}$ Ashworth scale: no increase in muscle tone (scale 1 ); slight increase in tone giving a 'catch' when affected part is moved in flexion or extension (scale 2); more marked increase in tone, but affected part is easily flexed (scale 3); considerable increase in tone and difficult passive movement (scale 4); affected part is rigid in flexion or extension (scale 5).

-: absent; +: mild; ++: moderate; +++: severe; F: female; M: male

study, conducted on the members of this family, was approved by the ethics committee of the Shandong University School of Medicine. Signed, informed consent was obtained from all participants prior to the study. Based on Harding's criteria, ${ }^{(1)}$ an experienced neurologist performed neurological examinations on 11 family members and one of the spouses. The index patient (patient II10) underwent electromyography, magnetic resonance imaging, neurophysiological studies, cerebrospinal fluid examination and cognitive tests. Other related neural diseases were excluded. The clinical features of patients with this pedigree, who might represent individuals with a pure form of HSP, are presented in Table I. Blood samples were obtained from 11 family members (symptomatic $n=4$; asymptomatic $n=7$ ) and one spouse (unaffected). Leucocyte genomic DNA was extracted using standard techniques.

We first tested whether HSP in the family was caused by a mutation in any of the known HSP loci. As SPG4 is the most common causative gene for ADHSP and SPG 4 mutation accounts for approximately $40 \%$ of AD pedigrees, ${ }^{(9)}$ we selected D2S2351 and D2S2347 (lying 0.2 Mb upstream and 0.7 Mb downstream of SPG4, respectively), two microsatellite markers with high heterozygosity in the population according to Marshfield genetic maps, for analysis. ${ }^{(18)}$ Polymerase chain reaction (PCR) amplification was performed in a $20 \mu \mathrm{l}$ volume containing $8 \mu \mathrm{l}$ of water, $4 \mu \mathrm{l}$ of $5 \times$ TNK50, $4 \mu$ l of $5 \times$ deoxyribonucleotide triphosphates, $0.5 \mu \mathrm{l}$ of each primer $(10 \mu \mathrm{M}), 2 \mu \mathrm{l}$ of genomic DNA (40 ng/ $\mu \mathrm{l}$ ) and $1 \mu \mathrm{l}$ of Taq DNA polymerase $(1 \mathrm{U} / \mu \mathrm{l}$; TianGen Biotech, Beijing, China), with an initial denaturation step of $5 \mathrm{~min}$ at $95^{\circ} \mathrm{C}$ to activate the polymerase, followed by 35 cycles of $95^{\circ} \mathrm{C}$ for $40 \mathrm{~s}, 55^{\circ} \mathrm{C}$ for $40 \mathrm{~s}, 72^{\circ} \mathrm{C}$ for $40 \mathrm{~s}$ and $\mathrm{a}$ final elongation at $72^{\circ} \mathrm{C}$ for $10 \mathrm{~min}$. Predicted amplicon sizes were confirmed by agarose gel electrophoresis. The amplified fragments of D2S2351 and D2S2347 (patients II3, II5, II8, II10 and III1), with internal size standards, were 201-282 bp. Polymorphism was estimated using polyacrylamide gel electrophoresis (PAGE).

The primers used were designed to examine entire exonic sequences of SPAST (MIM 604277) via PCR amplification and direct sequencing. Large exons were divided into several overlapping PCR fragments. From the index patient's sample, all exons, including exon-intron boundaries, were amplified by PCR and direct sequencing. The sequences of the other family members and 50 normal, unaffected individuals who were matched for geographical ancestry were confirmed by Sanger sequencing. Changes in exons were confirmed by repeat PCR and sequencing of both strands of the resulting product in ten family members other than the index patient and the spouse. The amplified fragments of exon 15, with internal size standards, were $373 \mathrm{bp}$ with the use of the designed primers. Polymorphism in the amplified fragments of exon 15 of the SPAST gene was estimated in four panels by non-denatured PAGE (ND-PAGE) for the 11 family members. Amplified products with mutations were ligated to a pMD-18T vector and transformed into Escherichia coli (E. coli) bacteria. Detected changes in the exons were precisely defined by subcloning PCR and sequencing.

\section{RESULTS}

The phenotype of the four patients (patients II3, II8, III0 and III1) who were found to have HSP was late disease onset (age $\geq 45$ years) and severe clinical manifestations. Three patients (patients II3, II10 and III1) had severe symptoms, while patient II8 showed severe symptoms that were relatively fewer in number. Symptoms in the proband (patient II10), a 69-year-old woman, began at the age of 45 years, and included progressively spastic gait, walking difficulty that required assistance, tendon 
hyperreflexia of the lower limbs and Babinski's sign. Initial symptoms of walking difficulty and progressively spastic gait in four of our affected patients began at a similar age. The disease progressed slowly, and there was correlation of clinical severity with disease duration. None of the patients exhibited upper extremity features or urinary disturbances (urgency and/or incontinence). Disease onset in patient III1 began at the age of 46 years, with mild walking difficulty, increasingly reduced muscle tone in the lower limbs and hyperreflexia. Patients III6 and IV1 were asymptomatic and showed no abnormal features on neurological examination at the ages of 42 and 23 years, respectively. As most of the third/fourth generation family members had not yet reached the age of disease onset, we could not determine whether they were affected or not based on phenotype alone. The family members available for linkage analysis were thus fewer and we were unable to get a high logarithm of odds (LOD) score at D2S2347. We found that among the available family members, alleles of D2S2347 cosegregated with the phenotype. Although we could not confirm that SPG 4 was the disease gene, we also could not exclude the possibility of it being the causative gene. There was no D2S2351 polymorphism found in the family.

Via Sanger sequencing, we were able to determine whether an SPG4 mutation was present in members of the Chinese family with ADHSP. Our results revealed a 14-bp heterozygous deletion (c.1630-1643delTACTCAGGAAGTGA) in exon 15 of SPAST, leading to a frameshift mutation and premature termination of translation (p.tyr544profsX28). The 14-bp heterozygous deletion was precisely defined by subcloning PCR products in E. coli and sequencing (Fig. 2). Our findings also identified cosegregation of the deletion and phenotype in other affected family members. This mutation was, however, found to be absent in 50 normal, unaffected individuals who were matched for geographical ancestry. Sequence analysis revealed that two patients (patients III6 and IV1 aged 42 and 23 years, respectively) carried the mutated gene with the 14-bp heterozygous deletion, although they were both asymptomatic and showed no abnormal features on neurological examination. Results of the ND-PAGE of the amplified fragments of exon 15 of SPAST showed that the affected family members (patients II3, II8, II10 and III1) carried a 14-bp deletion gene, as did two other patients who were asymptomatic (patients III6 and IV1), but those of the 50 normal, unaffected individuals who were matched for geographical ancestry were homozygous with no mutation.

\section{DISCUSSION}

In this study, combined linkage analysis and sequencing enabled the detection of a novel mutation in the SPAST gene that was causing ADHSP in a four-generation Chinese family. Previous reports have shown that HSP, which has a strong genetic basis, is clinically heterogeneous. The age of disease onset ranges from 1-77 years and the severity of symptoms differs greatly in many families with ADHSP. ${ }^{(8)}$ In the four-generation Chinese family

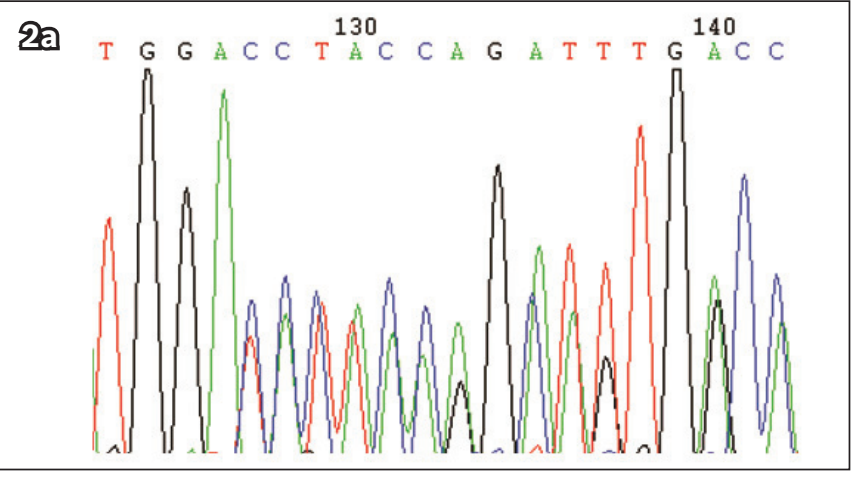

$2 b$

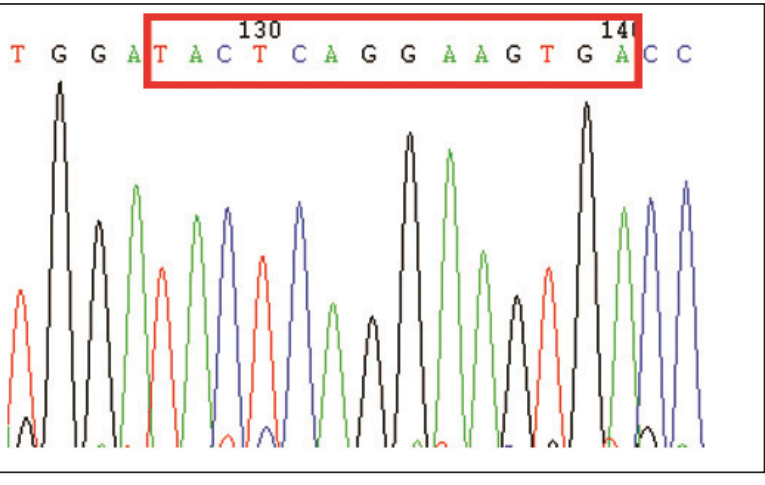

$2 \mathrm{C}$

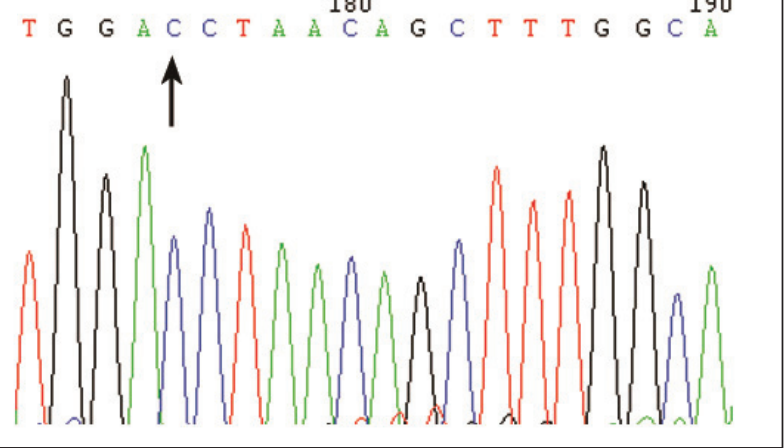

Fig. 2 A novel mutation was identified in a Chinese family with autosomal dominant hereditary spastic paraplegia. (a) The mutant sequence in exon 15 of SPAST among affected family members. (b) Normal sequence found in exon 15 of SPAST in an unaffected family member. (c) Site of the deletion mutation (arrow) found in exon 15 of SPAST in the index family member (patient II10), as defined upon sequencing of the subcloned polymerase chain reaction products.

with ADHSP studied here, the phenotypes of the four affected patients were late disease onset ( $\geq 45$ years) and severe clinical manifestations (with no upper extremity features or urinary disturbances - urgency and/or incontinence), although bladder disturbances were previously reported in approximately $50 \%$ of affected individuals. ${ }^{(19,20)}$

Most of the SPAST mutations reported include missense, nonsense or splice site mutations, small insertions or deletions, and large duplications and deletions. ${ }^{(11-14)}$ In our study, we detected a novel 14-bp heterozygous deletion (c.16301643delTACTCAGGAAGTGA) in exon 15 of the SPAST gene, which led to a frameshift mutation with a premature stop codon. However, this mutation was found to be absent in 50 normal individuals who were matched for geographical ancestry. Sequence analysis revealed the presence of the 14-bp deletion 
in two asymptomatic patients (patients III6 and IV1) who showed no abnormal features on neurological examination at age 42 years and 23 years, respectively. We could not determine whether incomplete dominance existed in this four-generation Chinese family with ADHSP, as the phenomenon's penetrance might be age-dependent or incomplete, or manifest subtle symptoms. ${ }^{(8,9)}$ Subtle symptoms may progress slowly and go unnoticed for years. The variation seen in exon 15 was at a highly conserved position, which predicted functional impact and cosegregation with the phenotype. Mutation (p.tyr544profsX28) between amino acids 544 and 571, with a premature termination, is predicted to disrupt the highly conserved functional domain (the AAA cassette), which includes the ATP binding and hydrolysis sites. ${ }^{(21)}$ The likely pathogenic mechanism for the frameshift mutation in our study is haploinsufficiency, which leads to unstable mRNA levels and absence or low level of expression of proteins. (22) Although haploinsufficiency (loss-of-function mechanism) is currently the most accepted view, gain-of-function of a 'toxic' or dominant negative activity could also partly explain this pathogenic mechanism of SPAST mutation. ${ }^{(15,16,23,24)}$ Hence, the mechanism of HSP (via mutation in SPG4) is still a subject of much dispute.

In conclusion, we investigated the SPAST gene via linkage analysis and sequencing in a four-generation Chinese family who exhibited ADHSP. We found a novel mutation in exon 15 of the SPG4 gene in members of this family, which was absent in unaffected individuals who were matched for geographical ancestry. The mutation in a highly conserved AAA cassette spanning exon 7 to 16 was predicted to have functional impact and found to completely cosegregate with the HSP phenotype. Our result expands the mutation spectrum of SPG4. The identification of this novel deletion in SPAST provides an opportunity to further study and increase our understanding of the mechanism of axonal neurodegeneration in HSP, which may have important therapeutic implications. As better understanding of the function of spasticity is important, further phenotypegenotype correlation studies will be required to help explain the variable expression of the SPG4 form for the prevention, diagnosis and treatment of HSP.

\section{ACKNOWLEDGEMENTS}

We thank the patients and their families for their participation. This work was supported by grants from the National Natural Science Foundation of China (grant no. 81072452) and the Research Award Fund for Outstanding Young Scientist of Shandong Province (2007BS0327).

\section{REFERENCES}

1. Harding AE. Classification of the hereditary ataxias and paraplegias. Lancet 1983; 1:1151-5.

2. Depienne C, Stevanin G, Brice A, Durr A. Hereditary spastic paraplegias: an update. Curr Opin Neurol 2007; 20:674-80.

3. Stevanin G, Ruberg M, Brice A. Recent advances in the genetics of spastic paraplegias. Curr Neurol Neurosci Rep 2008; 8:198-210.

4. Salinas S, Proukakis C, Crosby A, Warner TT. Hereditary spastic paraplegia: clinical features and pathogenetic mechanisms. Lancet Neurol 2008; 7:1127-38.

5. Slabicki M, Theis M, Krastev DB, et al. A genome-scale DNA repair RNAi screen identifies SPG48 as a novel gene associated with hereditary spastic paraplegia. PLoS Biol 2010; 8:e1000408.

6. Fink JK. Hereditary spastic paraplegia. Curr Neurol Neurosci Rep 2006; 6:65-76.

7. Lin P, Li J, Liu Q, et al. A missense mutation in SLC33A1, which encodes the acetyl-CoA transporter, causes autosomal-dominant spastic paraplegia (SPG42). Am J Hum Genet 2008; 83:752-9.

8. Meijer IA, Hand CK, Cossette P, Figlewicz DA, Rouleau GA. Spectrum of SPG4 mutations in a large collection of North American families with hereditary spastic paraplegia. Arch Neurol 2002; 59:281-6.

9. Alvarez V, Sánchez-Ferrero E, Beetz C, et al. Mutational spectrum of the SPG4 (SPAST) and SPG3A (ATL1) genes in Spanish patients with hereditary spastic paraplegia. BMC Neurol 2010; 10:89.

10. The Human Gene Mutation Database [online]. Available at: www.hgmd. cf.ac.uk/ac/index.php. Accessed November 15, 2012.

11. Beetz C, Nygren AO, Schickel J, et al. High frequency of partial SPAST deletions in autosomal dominant hereditary spastic paraplegia. Neurology 2006; 67:1926-30.

12. Depienne C, Fedirko E, Forlani S, et al. Exon deletions of SPG4 are a frequent cause of hereditary spastic paraplegia. J Med Genet 2007; 44:281-4.

13. Erichsen AK, Inderhaug E, Mattingsdal M, Eiklid K, Tallaksen CM. Seven novel mutations and four exon deletions in a collection of Norwegian patients with SPG4 hereditary spastic paraplegia. Eur J Neurol 2007; 14:809-14.

14. Mitne-Neto M, Kok F, Beetz C, et al. A multi-exonic SPG4 duplication underlies sex-dependent penetrance of hereditary spastic paraplegia in a large Brazilian pedigree. Eur J Hum Genet 2007; 15:1276-9.

15. Solowska JM, Garbern JY, Baas PW. Evaluation of loss of function as an explanation for SPG4-based hereditary spastic paraplegia. Hum Mol Genet 2010; 19:2767-79.

16. Errico A, Ballabio A, Rugarli El. Spastin, the protein mutated in autosomal dominant hereditary spastic paraplegia, is involved in microtubule dynamics. Hum Mol Genet 2002; 11:153-63.

17. Park SH, Zhu PP, Parker RL, Blackstone C. Hereditary spastic paraplegia proteins REEP1, spastin, and atlastin-1 coordinate microtubule interactions with the tubular ER network. J Clin Invest 2010; 120:1097-110.

18. Mammalian Genotyping Service. Comprehensive human genetics maps (online). Available at: research.marshfieldclinic.org/genetics/ GeneticResearch/compMaps.asp. Accessed November 14, 2012.

19. Harding AE. Hereditary "pure" spastic paraplegia: a clinical and genetic study of 22 families. J Neurol Neurosurg Psychiatry 1981; 44:871-83.

20. Harding AE. Hereditary spastic paraplegias. Semin Neurol 1993; 13:333-6.

21. Hazan J, Fonknechten N, Mavel D, et al. Spastin, a new AAA protein, is altered in the most frequent form of autosomal dominant spastic paraplegia. Nat Genet 1999; 23:296-303.

22. Fonknechten N, Mavel D, Byrne P, et al. Spectrum of SPG4 mutations in autosomal dominant spastic paraplegia. Hum Mol Genet 2000; 9:637-44.

23. Patrono C, Casali C, Tessa A, et al. Missense and splice site mutations in SPG4 suggest loss-of-function in dominant spastic paraplegia. J Neurol 2002; 249:200-5.

24. Charvin D, Cifuentes-Diaz C, Fonknechten N, et al. Mutations of SPG4 are responsible for a loss of function of spastin, an abundant neuronal protein localized in the nucleus. Hum Mol Genet 2003; 12:71-8. 